\title{
Comparing numerical error and visual quality in reconstructions from compressed digital holograms
}

\author{
Taina M. Lehtimäki, ${ }^{a}$ Kirsti Sääskilahti, ${ }^{a}$ Tomi Pitkäaho, ${ }^{a}$ and Thomas J. Naughton ${ }^{\text {ba }}$ \\ ${ }^{a}$ University of Oulu, Oulu Southern Institute, Vierimaantie 5, 84100 Ylivieska, Finland \\ ${ }^{\mathrm{b}}$ Department of Computer Science, National University of Ireland Maynooth, Ireland
}

\begin{abstract}
Digital holography is a well-known technique for both sensing and displaying real-world three-dimensional objects. Compression of digital holograms has been studied extensively, and the errors introduced by lossy compression are routinely evaluated in a reconstruction domain. Mean-square error predominates in the evaluation of reconstruction quality. However, it is not known how well this metric corresponds to what a viewer would regard as perceived error, nor how consistently it functions across different holograms and different viewers. In this study, we evaluate how each of seventeen viewers compared the visual quality of compressed and uncompressed holograms' reconstructions. Holograms from five different three-dimensional objects were used in the study, captured using a phase-shift digital holography setup. We applied two different lossy compression techniques to the complex-valued hologram pixels: uniform quantization, and removal and quantization of the Fourier coefficients, and used seven different compression levels with each.
\end{abstract}

Keywords: image compression, perceived quality, noise, digital holography, three-dimensional imaging

\section{INTRODUCTION}

Holograms are a natural way of encoding three-dimensional (3D) information about a real-world scene ${ }^{1}$. Digital holograms ${ }^{2-7}$ are created using a laser and a digital camera that using the principle of interference records the intensity and phase of the light scattered from the 3D object(s). These digital holograms contain 3D data, which are typically visualized with two-dimensional (2D) reconstructions from different viewing angles and with different in-focus depths. Three-dimensional optical displays are not yet commonplace for digital holograms.

As with any type of media, compression is a vital component of any digital hologram capture and display system. The challenges in compressing digital holograms of macroscopic 3D scenes are that the holograms contain a Fresnel transformed version of the scene rather than an image of the scene, the holograms and their reconstructions are corrupted by speckle, ${ }^{8}$ and the reconstructed 3D image (and possibly the hologram itself) are complex-valued. Quantization and compression of digital holograms has been studied previously, ${ }^{7,-16}$ and the errors introduced by lossy compression are routinely evaluated numerically in one reconstruction domain. Mean-square error predominates in the evaluation of reconstruction quality. However, it is not known how well this metric corresponds to what a viewer would regard as perceived error, nor how consistently it functions across different holograms and different viewers.

Besides numerical evaluation methods, perceptual evaluation is very important and widely used way of comparing image compression algorithms. An extensive review of quality metrics and psychophysical testing techniques that are typically used in still image compression studies can be found in the article written by Eckert and Bradley. ${ }^{17}$ Subjective perceptual evaluation is often considered as most reliable way of assessing the quality of image and video. The goal of objective image and video quality assessment research is to design quality metrics that can predict perceived image and video quality automatically. Perceptual evaluation can be used to monitor image quality, to benchmark, and to optimize the algorithms and the parameter settings. ${ }^{18}$

The purpose of this study is to find out how numerical evaluation and visual evaluation differ and support each other for compression of digital holographic data. In this study, we evaluate how each of seventeen viewers compared the visual quality of compressed and uncompressed holograms' reconstructions. These responses were plotted against numerical error. Viewers were asked to determine if two reconstructions were the same quality or which was lower quality. They

\footnotetext{
Three-Dimensional Imaging, Visualization, and Display 2010 and Display Technologies and Applications for Defense,

Security, and Avionics IV, edited by Bahram Javidi, Jung-Young Son, John Tudor Thomas, Daniel D. Desjardins,

Proc. of SPIE Vol. 7690, 769012 · (C) 2010 SPIE · CCC code: 0277-786X/10/\$18 · doi: 10.1117/12.853344
} 


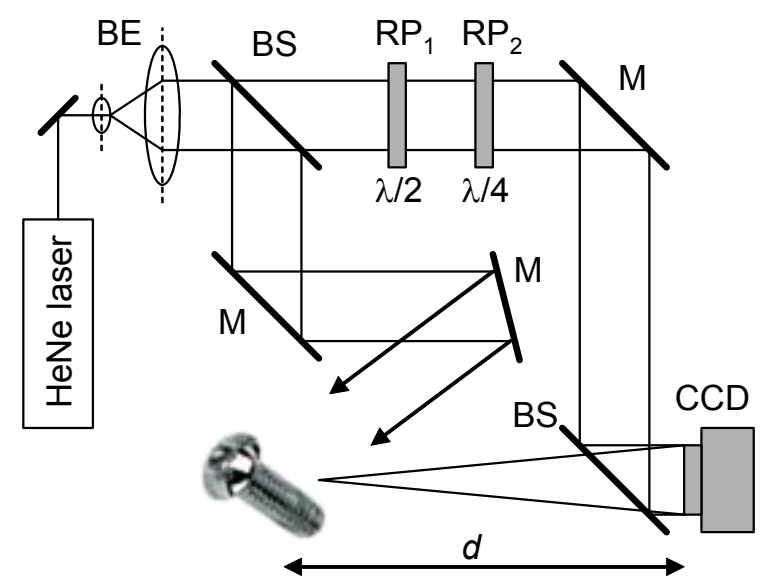

Figure 1. Optical apparatus for phase shift digital holography: BE, beam expander; BS, beamsplitter; M, mirror; RP, retardation plate; $\mathrm{CCD}$, digital camera.

were also asked to determine if quality was so low that it was not possible to tell what was pictured in the image. From this we determined what error level corresponded to visually lossless compression. Holograms from five different threedimensional objects were used in the study, captured using a phase-shift digital holography setup. We applied two different lossy compression techniques to the complex-valued hologram pixels: uniform quantization (QNT), and uniform quantization of the Fourier coefficients (DFT), and used seven different compression levels with each.

The manuscript is organized as follows. In Sect. 2 we describe the methods, including hologram capture, compression and numerical evaluation techniques, stimuli, and procedure. We present the results in Sect. 3 and conclude in Section 4.

\section{METHODS}

\subsection{Hologram capture}

Our digital holograms are recorded using the optical setup (shown in Fig. 1) based on a Mach-Zehnder interferometer architecture in an in-line configuration. A spatially filtered linearly polarized helium neon $(632.8 \mathrm{~nm})$ laser beam is split into object and reference beams, both of which are spatially filtered and expanded. The first beam illuminates the 3D object placed at a distance $d$ from a 10-bit 2032 $\times 2048$ pixel CCD camera. The reference beam passes through half-wave plate RP1 and quarter-wave plate RP2. Through permutation of the fast and slow axes of the plates we can achieve phase shifts of $0,-\pi / 2,-\pi$, and $-3 \pi / 2$. The reference beam combines with the light diffracted from the object and forms an interference pattern in the plane of the camera. At each of the four phase shifts we record an interferogram. Using these four intensity images, the complex-valued camera-plane wavefront can be approximated in a computer to good accuracy using phase-shift interferometry. ${ }^{19,20}$ Digital holograms of reasonably diffuse macroscopic 3D objects were used in the experiments. The objects had approximate dimensions of $5 \mathrm{~mm} \times 5 \mathrm{~mm} \times 5 \mathrm{~mm}$ and were positioned approximately $d=$ $350 \mathrm{~mm}$ from the camera.

\subsection{Compression, reconstruction, and numerical evaluation}

Holograms contained $2032 \times 2048$ pixels when captured by CCD camera and were increased to $2048 \times 2048$ pixels in size after padding with a few blank columns. Two hologram-domain compression algorithms were employed in this study: quantization and Fourier coefficient quantization.

Quantization (QNT) is a data reduction technique that encodes the set of input data values using an appropriate subset of values. Uniform quantization is a data-dependent technique and is the optimal choice when the data values are uniformly distributed. Since our hologram data consists of unevenly distributed complex values, the set of quantization values should be selected carefully in order to account for these biases. When the set of quantization values is a nonuniform 
sampling of the input data, the quantization is referred to as nonuniform quantization. For simplicity, we have chosen uniform quantization in this study. Given a hologram $H(x, y)$, the rescale and quantisation step for each pixel is defined as

$$
H^{\prime}(x, y)=\operatorname{round}\left[H(x, y) \sigma^{-1} \gamma y^{-1},\right.
$$

where $\gamma$ is the number of uniform quantisation levels required per real and imaginary value, and

$$
\sigma=\max \{|\min [\operatorname{Im}(H)],| \max [\operatorname{Im}(H)],|\min [\operatorname{Re}(H)],| \max [\operatorname{Re}(H)]\} .
$$

Here, $\max (\cdot)$ returns the maximum scalar of its arguments, $|\cdot|$ denotes absolute value, $\operatorname{Re}(\cdot)$ and $\operatorname{Im}(\cdot)$ return the real and imaginary component of a complex argument, respectively, and round $(\cdot)$ maps to the closest integer.

The discrete Fourier transform (DFT) is applied to each non-overlapping $8 \times 8$ pixel blocks of the hologram. From the resulting 64 complex-valued coefficients in each block, the $(64-p)$ coefficients with the lowest amplitude are set to zero. In case of a choice between two coefficients of equal amplitude, the one with the lower phase value is set to zero. (Although in many respects an arbitrary decision, is ensures repeatability in the calculations.) The hologram remains in its piecewise discrete Fourier transformed state, and the remaining non-zero Fourier coefficients are quantized to 4 bits.

Neither quantization algorithm will reduce the data size on their own, of course. A lossless compression stage would be required to complete the compression process, and would be needed if one wished to compare perceived error to compression ratio.

To evaluate numerical error, each nonoverlapping $8 \times 8$ pixel block was inverse discrete Fourier transformed in the case of DFT quantization (no step was necessary in the case of QNT quantization) and the hologram reconstructed. Reconstructions from holograms were obtained using a discrete Fresnel transform at a reconstruction distance that was judged to bring most of the object into focus.

Speckle reduction was employed to more accurately measure the numerical error due to hologram compression; small modifications to digital holograms can give rise to different speckle patterns and it was deemed necessary to weight less the influence of the speckle pattern defects in the numerical error calculation compared to the influence of the underlying object defects. For these experiments we used two approaches, choosing the approach that gave more visually appealing speckle reduced images in each case: we used mean filtering ${ }^{11}$ for hologram no. 2 and Fourier filtering ${ }^{21}$ for each of the other holograms. For mean filtering, we applied a $3 \times 3$ pixel mean filter to the reconstructed amplitude and then subsampled the hologram to half its height and width (summing the values in each $2 \times 2$ pixel block to get the value for each new pixel). For Fourier filtering, we applied a window size of $512 \times 512$ pixels (for Holograms 1 and 4 ) or $256 \times 256$ pixels (for Holograms 3 and 5) and then subsampled the hologram to half its height and width (summing the values in each $2 \times 2$ pixel block to get the value for each new pixel). Subsampling was necessary to fit two reconstructions side by side into the $1024 \times 768$ pixel $2 \mathrm{D}$ display.

The final step was to crop (consistently) the region of interest from each hologram reconstruction and normalize the intensity so that the mean pixel intensity in each reconstruction was 1.

The mean error per pixel was then calculated between this reconstruction $R^{\prime}$ and a reconstruction $R$ from the original hologram processed in an identical way (but without the quantization) using

$$
\operatorname{err}=\frac{\sum_{y=1}^{N} \sum_{x=1}^{M}\left|R(x, y)-R^{\prime}(x, y)\right|}{M N}
$$

where $M$ and $N$ are the height and width, respectively, of the region of interest. Images were then stretched to an 8-bit grayscale colormap and written to disk to be recalled later for the creation of stimuli. 


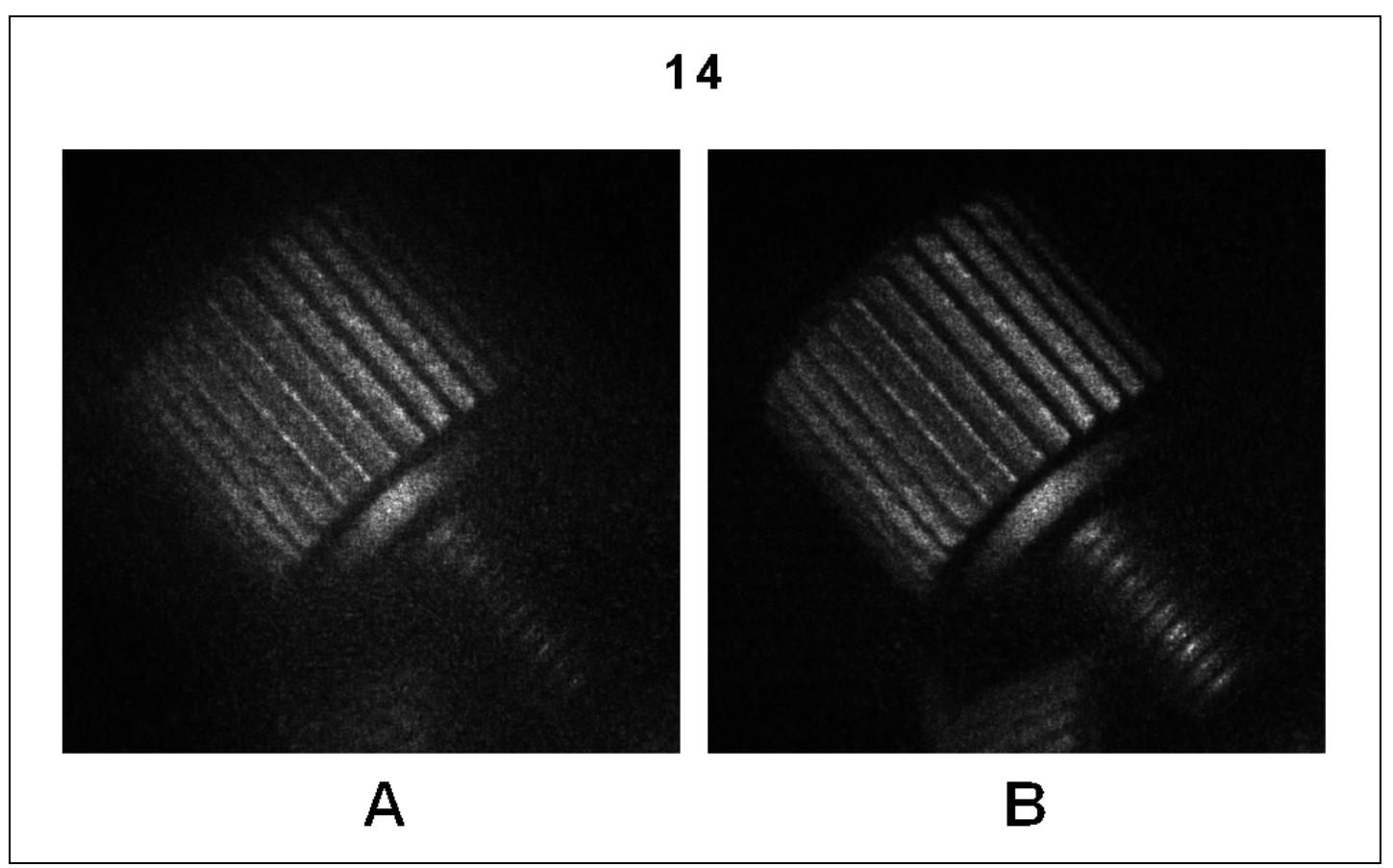

Figure 2. Example of the stimuli shown to the test subjects: Hologram 1, compression method DFT, compression level 6. On the left $\mathrm{A}$ is the reconstruction taken from compressed hologram and on the right $\mathrm{B}$ the original reconstruction. On the top the unique stimulus number (14) was displayed.

\subsection{Subjects and apparatus}

17 subjects participated in this study. Their ages were between 25 and 53, average 34. Eight of them were female and 9 male. For displaying the stimuli we used regular PC and 15 inch LCD display.

\subsection{Stimuli}

A total of 72 reconstruction pairs were used as stimuli consisting of:

- 5 holograms

- 2 quantization algorithms QNT and DFT

- 7 quantization levels ( 8 in the case of DFT quantization for holograms 4 and 5, for better numerical error coverage)

The seven levels for QNT corresponded to numbers of quantizations levels in each real and imaginary value from the set $\{31,15,11,9,7,5,3\}$. The seven levels for DFT corresponded to numbers of values of $p$ from the set $\{20,15,10,7,5$, $3,1\}$ with 25 as the extra level for holograms 4 and 5 .

An example of the displayed stimuli is in Figure 2. On the top there is the stimulus number and at the bottom A and B denoting the two reconstructions. Stimuli were $941 \times 630$ pixels in size, which contained two $450 \times 450$ reconstructions (the regions of interest mentioned earlier). When stimuli were displayed on the screen, it was found that not all were bright enough for comfortable viewing, and a brighten threshold was applied where necessary. This was applied as follows. When the intensity values of the reconstruction were rescaled to the range $[0,1]$, all values greater than the threshold were set to the threshold value. The intensity values were then rescaled back to the full dynamic range of the display. Hologram 5 needed a brighten threshold of 0.9 . 


\section{DFT compressed lower quality than original}

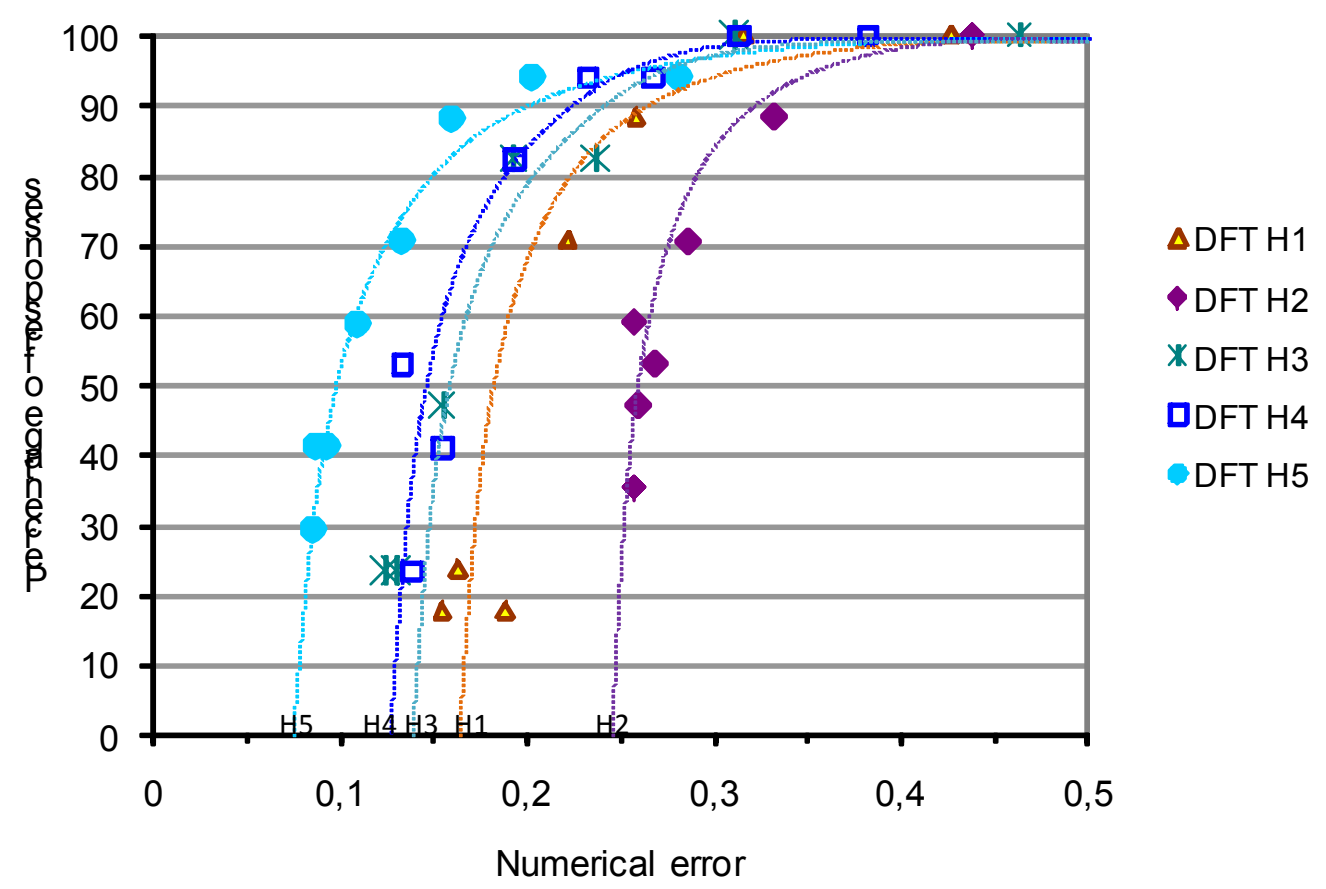

Figure 3. Result for DFT algorithm for each five holograms for perceived lower quality in the compressed reconstruction. On the horizontal axis is the numerical error and on the vertical axis the percentage of responses. Trendlines are fitted for easier comparison between holograms.

\subsection{Procedure}

Subjects were shown in total 72 reconstruction pairs. Stimuli were shown in random order. Within stimuli the side A or $\mathrm{B}$ of the compressed and original image was also randomized. Subjects were asked to fill in a questionnaire sheet with stimuli numbers prefilled in a table. They were told that they participate in evaluation of compression methods of digital holograms. They were asked to decide if the two images displayed were either same quality or which of the two A or B was lower quality. They were to write $\mathrm{S}$ if the quality was the same or either A or B for the lower quality image. Also they were instructed to write a "-." if it was not possible to recognize what was in the lower quality image without the aid from the original image.

\section{RESULTS}

\subsection{Perceived lower quality in the compressed reconstruction}

In the Figures 3 and 4 we show results for the DFT and QNT algorithm for each five holograms for perceived lower quality in the compressed reconstruction.

In Figure 3 we can see that the plot of probability of perceived defects (noticeable difference) over numerical error is not linear: in fact it more closely resembles a logarithmic function. At low compression ratios (which we equate with a low probability of noticeable defects), even a small change in numerical error results big difference in percentage of responses. At high compression ratios, fewer respondents could differentiate hologram reconstructions with different amounts of defects. Although this trend is evident in every hologram, the absolute results are hologram specific. For 


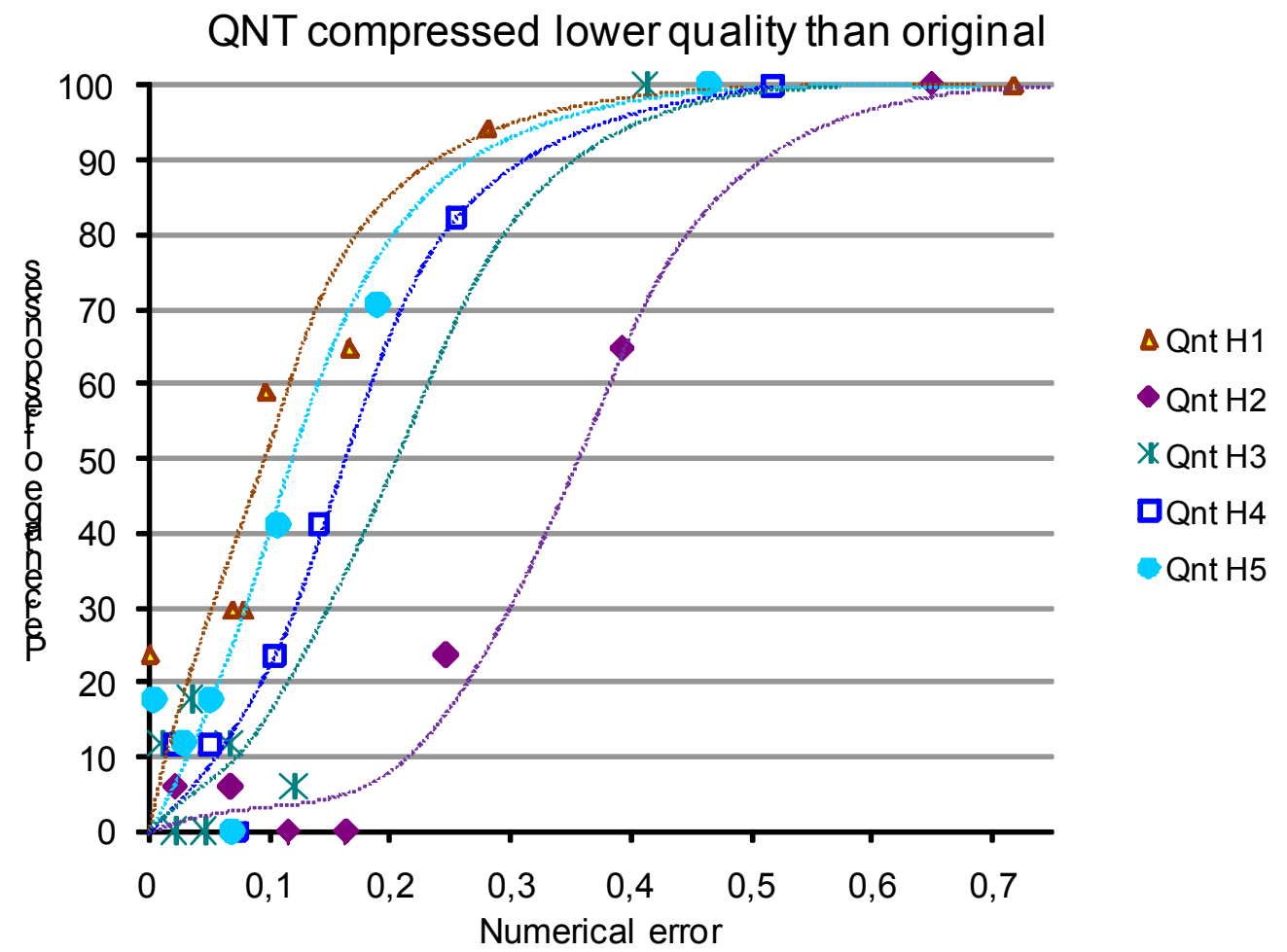

Figure 4. Result for QNT algorithm for each of the five holograms for perceived lower quality in the compressed reconstruction. On the horizontal axis is the numerical error and on the vertical axis the percentage of responses. Trendlines are fitted for easier comparison between holograms.

example, $30 \%$ of subjects noticed difference for hologram 5 compressed with 0.09 numerical error and about same percentage of responses for hologram 2 denotes to 0.26 numerical error. This indicates that when evaluating hologram compression algorithms, the absolute numerical reconstructed error will not be as useful if given in isolation. For a numerical error of 0.15 , approximately half of the respondents did not notice defects in approximately half of the compressed holograms. Even with smallest amount of compression the difference between original and compressed image was noticed by $20 \%$ to $35 \%$ of the responses depending on the hologram. This shows that our compression strategy is too coarse grained to evaluate what numerical error is not noticed by $90 \%$ of respondents over $90 \%$ of holograms, for example.

In Figure 4 for the QNT algorithm for perceived lower quality in the compressed reconstruction it can be seen that even small change in numerical error results big difference in percentage of responses. With smallest amount of compression the difference between original and compressed image was not always noticed e.g. 0.16 numerical error for Hologram 2 was not noticed by any of our test subjects. However for Hologram 1 even the smallest amount of compression was noticed by over $20 \%$ of our test subjects although this denotes to very small numerical error 0.002 . The responses are very dependent on the holograms also for this compression algorithm. However for this hologram results suggest that a generalization can be made that for less than 0.1 numerical error less than $20 \%$ of viewers will notice difference between original and compressed hologram. 


\section{DFT compression algorithm}

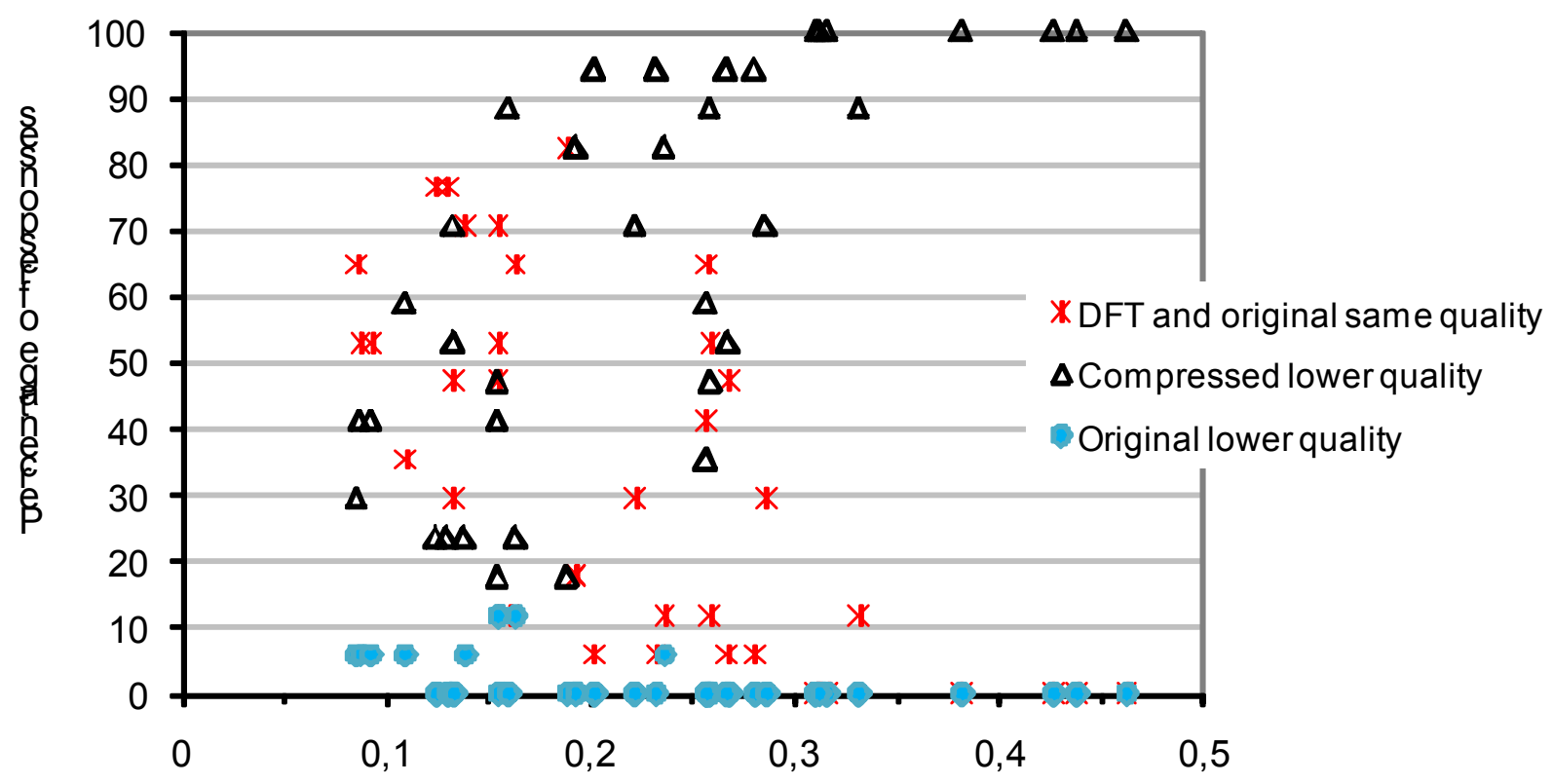

Numerical error

Figure 5. Complete comparison results for DFT algorithm. On the horizontal axis is the numerical error and on the vertical axis the percentage of responses. Results show the distribution of all response options for all holograms, and for all compression levels for each hologram. The three response options for comparing the quality of the compressed and original image were: same quality, compressed lower, and original lower.

\subsection{Complete comparison results}

In Figures 5 and 6 we present the complete comparison results for DFT and QNT algorithms respectively. Results for the DFT algorithm in Figure 5 show the distribution of all response options for all holograms, and for all seven compression ratios for each hologram. The three response options for comparing the quality of the compressed and original image were: same quality, compressed lower, or original lower. The results show that for high numerical error values (c. over 0,33 ) the compressed was always reported to be the lower quality one and for numerical error values up to 0,2 numerical error the responses for the same quality or compressed lower quality are quite equally typical. Original is reported to have poorer quality than compressed reconstruction in only in small number of cases.

In Figure 6 the results for the QNT algorithm show that for high numerical error values (c. over 0,4) the compressed was always reported to be the lower quality one and for numerical error values up to 0,15 numerical error the responses for the same quality are most typical. Original is reported to have poorer quality than the compressed reconstruction up to 30 $\%$ of the subjects, which is much more than for DFT algorithm. This is reported up to $30 \%$ of the subjects when numerical error is up to 0,11 and up to $18 \%$ of the subjects when numerical error is up to 0,25 . For larger numerical errors this phenomena disappears. From this we can draw a conclusion that for small amount of compression for some holograms the reconstruction can be perceived to have enhanced in quality. However this is true only by up to $30 \%$ of subjects. 


\section{QNT compression algorithm}

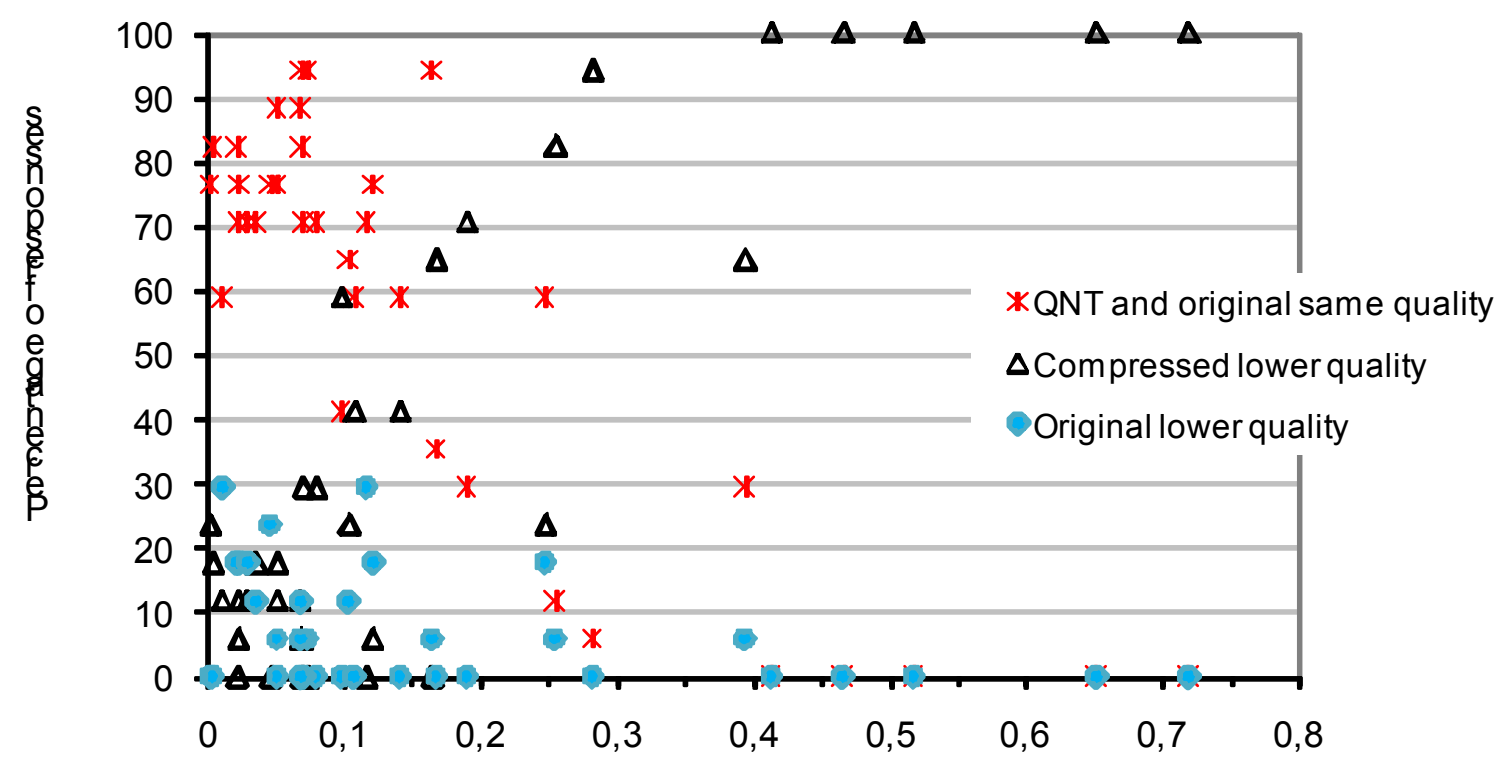

Numerical error

Figure 6. Complete comparison results for QNT algorithm. On the horizontal axis is the numerical error and on the vertical axis the percentage of responses. Results show the distribution of all response options for all holograms, and for all compression levels for each hologram. The three response options for comparing the quality of the compressed and original image were: same quality, compressed lower, and original lower.

\subsection{Compressed image not recognisable}

In Figures 7 and 8 we show results to the DFT and QNT algorithm for when compressed image was not recognisable for the each of the five holograms separately. For both algorithms it is clear that the result is depended on the hologram, however the dependency curve for different holograms have similar shape for both algorithms.

From Figure 7 for DFT algorithm we can determine that for $50 \%$ of the subjects loss of recognition of the object corresponds approximately between 0,2 and 0,5 numerical error for different holograms. In Figure 8 respectively for the QNT algorithm it can be seen that $50 \%$ of loss of recognition of the object varies approximately between 0,4 and 0,7 numerical error for different holograms. 


\section{DFT compressed image not recognisable}

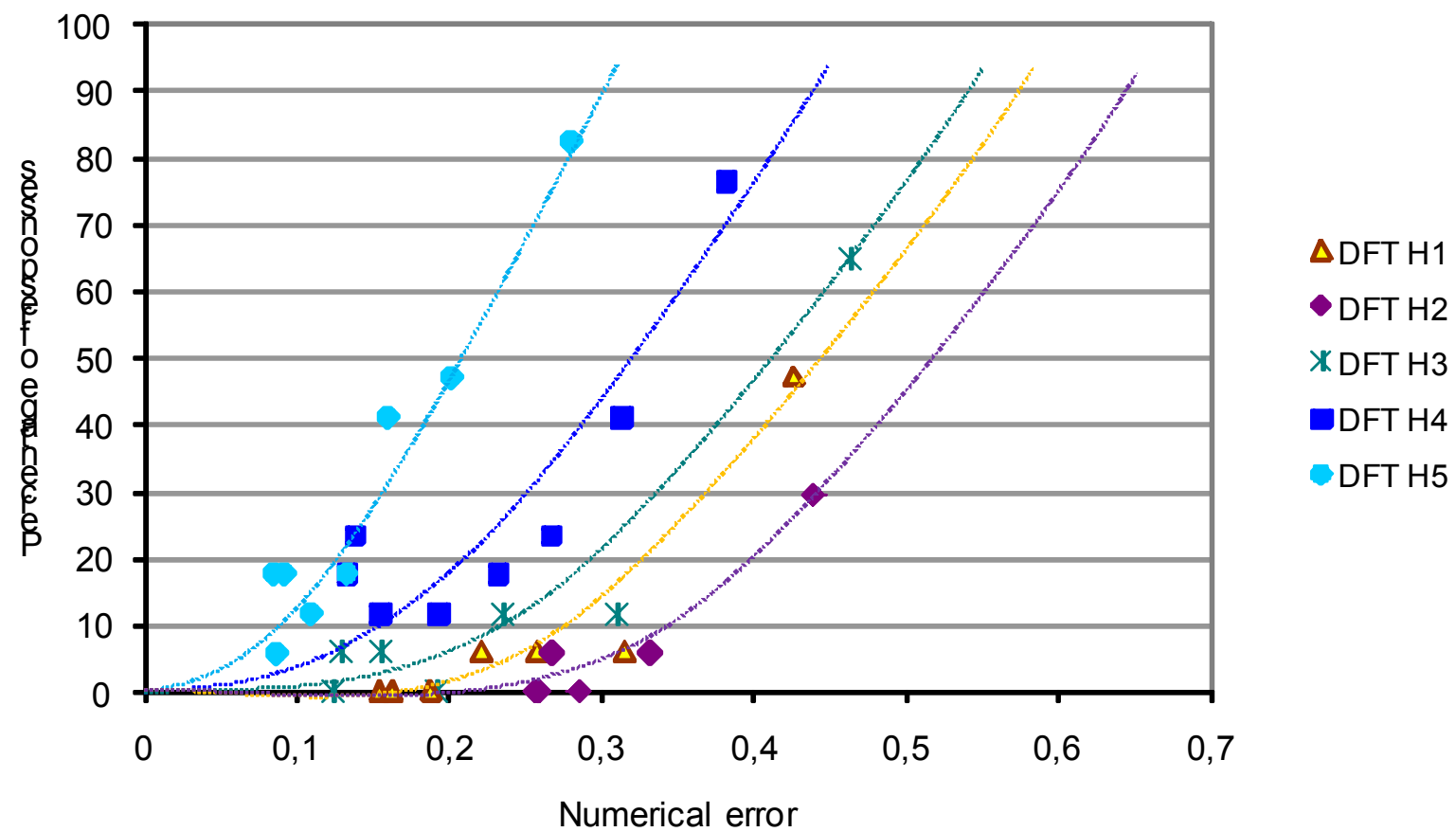

Figure 7. Compressed image is not recognisable responses for DFT algorithm. On horizontal axis the numerical error and on the vertical axis the percentage of responses for when the compressed image was not recognisable. The results are shown for each of the five holograms separately and trend lines are fitted for easier comparison.

\section{CONCLUSION}

The errors introduced by lossy compression of digital holograms are routinely evaluated numerically in a reconstruction domain. Mean-square error predominates in the evaluation of reconstruction quality. However, it is not known how well this metric corresponds to what a viewer would regard as perceived error, nor how consistently it functions across different holograms and different viewers. In this study, we evaluated how each of seventeen viewers compared the visual quality of compressed and uncompressed holograms' reconstructions. We applied two different lossy compression techniques to the complex-valued hologram pixels: uniform quantization, and removal and quantization of the Fourier coefficients, and used seven different compression levels with each. From these results it is possible to determine what numerical error level corresponds to visually lossless compression.

\section{ACKNOWLEDGEMENTS}

The research leading to these results has received funding from Science Foundation Ireland under the National Development Plan, the Academy of Finland, and the European Community's Seventh Framework Programme FP7/20072013 under Grant agreement No. 216105 (acronym "Real 3D"). We thank Conor Mc Elhinney for assistance with the hologram capture. 


\section{QNT compressed image not recognisable}

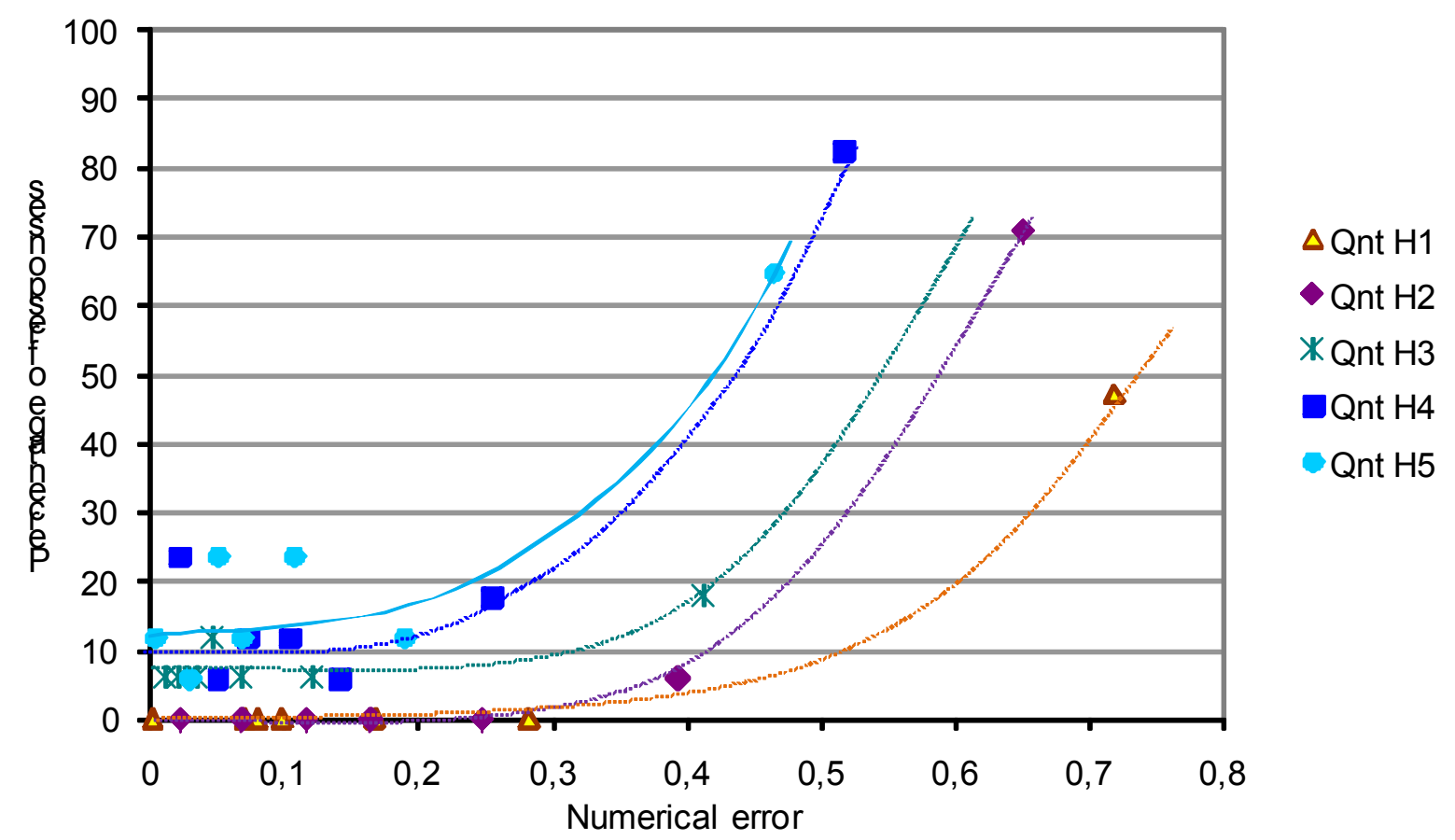

Figure 8. Compressed image is not recognizable responses for QNT algorithm. On horizontal axis the numerical error and on the horizontal axis the percentage of responses for when the compressed image was not recognizable. The results are shown for each of the five holograms and trend lines are fitted for easier comparison.

\section{REFERENCES}

[1] Gabor, D., "A new microscopic principle," Nature 161, 777-778 (1948).

[2] Goodman, J. W. and Lawrence, R. W., "Digital image formation from electronically detected holograms," Applied Physics Letters 11, 77-79 (1967).

[3] Kronod, M. A., Merzlyakov, N. S. and Yaroslavskii, L. P., "Reconstruction of a hologram with a computer," Sov. Phys. Tech. Phys 17, 333 (1972).

[4] Schnars, U. and Jueptner, W., [Digital Holography: Digital Hologram Recording, Numerical Reconstruction, and Related Techniques], Springer (2005).

[5] Kreis, Th., [Handbook of Holographic Interferometry: Optical and Digital Methods], Wiley (2005).

[6] Onural, L. and Scott, P. D., "Digital decoding of in-line holograms," Optical Engineering 26, 1124-1132 (1987).

[7] Frauel, Y., Naughton, T. J., Matoba, O., Tajahuerce, E. and Javidi, B., "Three-dimensional imaging and processing using computational holographic imaging," Proceedings of the IEEE 94, 636-653 (2006).

[8] J.C. Dainty, [Laser Speckle and Related Phenomena], Springer Verlag, Berlin, second edition (1984).

[9] Dallas, W. J. and Lohmann, A. W., "Phase quantization in holograms - depth effects," Appl. Opt. 11, 192-194 (1972).

[10] Naughton, T. J., Frauel, Y., Javidi, B. and Tajahuerce, E., "Compression of digital holograms for three-dimensional object reconstruction and recognition," Appl. Opt. 41, 4124-4132 (2002).

[11] Naughton, T. J., Mc. Donald, J. B. and Javidi, B., "Efficient compression of Fresnel fields for internet transmission of three-dimensional images," Appl. Opt. 42, 4758-4764 (2003).

[12] Nomura, T., Okazaki, A., Kameda, M., Morimoto, Y. and Javidi, B., "Image reconstruction from compressed encrypted digital hologram," Opt. Eng. 44, 075801 (2005).

[13] Kayser, D., Kreis, T. and Jüptner, W., "Compression of digital holographic data using its electromagnetic field properties,” Proc. SPIE 5908, 97-105 (2005). 
[14] Yamaguchi, I., Yamamoto, K., Mills, G. A. and Yokota, M., "Image reconstruction only by phase in phase-shifting digital holography," Appl. Opt. 45, 975-983 (2006).

[15] Darakis, E. and Soraghan, J. J., "Compression of interference patterns with application to phase-shifting digital holography," Appl. Opt. 45, 2437-2443 (2006).

[16] Shortt, A. E., Naughton, T. J., Javidi, B., "Histogram approaches for lossy compression of digital holograms of three-dimensional objects," IEEE Transactions on Image Processing 16, 1548-1556 (2007).

[17] Eckert, M. P. and Bradley, A. P., "Perceptual quality metrics applied to still image compression," Signal Processing 70, $177-200$ (1998).

[18] Wang, Z., Sheikh, H. R. and Bovik, A. C., "Objective video quality assessment," Chapter 41 in [The Handbook of Video Databases: Design and Applications], B. Furht and O. Marqure, ed., CRC Press, 1041-1078 (2003).

[19] Yamaguchi, I. and Zhang, T., "Phase-shifting digital holography," Optics Letters 22, 1268-1270 (1997).

[20] Frauel, Y., Tajahuerce, E., Castro, M. A. and Javidi B., "Distortion-tolerant 3D object recognition using digital holography," Applied Optics 40, 3887-3893 (2001).

[21] Maycock, J., Hennelly, B. M., McDonald, J. B., Frauel, Y., Castro, A., Javidi, B. and Naughton, T. J., "Reduction of speckle in digital holography by discrete Fourier filtering," Journal of the Optical Society of America A 24, 1617-1622 (2007). 